\title{
Characterization of Carotenoid Accumulation and Carotenogenic Gene Expression During Fruit Ripening in Red Colored Pulp of 'Siam Red Ruby' Pumelo (Citrus grandis) Cultivated in Thailand
}

\author{
Nopparat Tatmala ${ }^{1}$, Gang $\mathrm{Ma}^{2}$, Lancui Zhang ${ }^{2}$, Masaya Kato ${ }^{2}$ and Samak Kaewsuksaeng ${ }^{3 *}$ \\ 'Program of Biotechnology, Faculty of Technology and Community Development, Thaksin University, Phatthalung Campus, \\ Phatthalung 93210, Thailand \\ ${ }^{2}$ Department of Bioresource Sciences, Faculty of Agriculture, Shizuoka University, Shizuoka 422-8529, Japan \\ ${ }^{3}$ Department of Plant Science, Faculty of Technology and Community Development, Thaksin University, Phatthalung Campus, \\ Phatthalung 93210, Thailand
}

The 'Siam Red Ruby' (Citrus grandis) is a new lycopene-accumulating pumelo variety, which is grown only in the Pakpanang area of the Nakhon Si Thammarat province of Thailand. In this study, we harvested 'Siam Red Ruby' fruit at five ripening stages, and the changes in the accumulation of carotenoid and carotenogenic gene expression were investigated in the pulp. During the ripening process, the contents of phytoene, $\zeta$-Carotene, lycopene, $\beta$-carotene, $\beta$-cryptoxanthin, and all-trans-violaxanthin increased gradually, while the contents of $\alpha$ carotene and lutein decreased in the pulp of 'Siam Red Ruby'. In the mature fruit of 'Siam Red Ruby', a high amount of lycopene was accumulated in the pulp, accounting for more than $93.26 \%$ of the total carotenoid content. The massive accumulation of lycopene led to an attractive red color of the 'Siam Red Ruby' pulp. In addition, gene expression results showed that the increases in the expression of upstream genes (CitPSY, CitPDS, CitZDS, CitZISO, and CitCRTISO), and decreases in the expression of genes downstream (CitLCYb1, CitLCYb2, CitLCYe, CitHYb, and CitHYe) was the main mechanism of lycopene accumulation in the pulp of 'Siam Red Ruby' pumelo.

Key Words: carotenoid accumulation, gene expression, lycopene, ‘Siam Red Ruby’ pumelo.

\section{Introduction}

'Siam Red Ruby' pumelo (Citrus grandis) originated in the Pakpanang area of the Nakhon Si Thammarat province of Thailand. It is a geographical indication (GI) product of Thailand and is a popular pumelo cultivar in the premium fruit marketplace. The external appearance of the 'Siam Red Ruby' pumelo fruit is characterized by a dark green colored peel with soft hair and clear oil glands. The internal appearance of the 'Siam Red Ruby' pumelo fruit is characterized by a dark pink to red color and a sour-sweet taste (Kaewtubtim and Issarakraisila, 2011). Recently, demand for 'Siam Red Ruby' fruit has increased by $40 \%$

Received; September 26, 2019. Accepted; December 2, 2019.

First Published Online in J-STAGE on February 7, 2020.

This work was supported by the Thailand Research Fund under the Research and Researchers for Industries (RRI) to Nopparat Tatmala (PHD60I0076).

* Corresponding author (E-mail: samak@tsu.ac.th). in domestic, and $60 \%$ in international markets, especially markets in China, Taiwan, Hong Kong, Malaysia, Singapore, and Brunei. Today, commercial cultivation sites for 'Siam Red Ruby' pumelo are expanding, along with continuous plantation operations due to the high value and market demand (Department of Agriculture Extension, 2019). The 'Siam Red Ruby' pumelo is rich in bioactive compounds such as ascorbic acid (vitamin C), $\alpha$-tocopherol (vitamin E), and flavonoids (i.e., naringin, narirutin, hesperidin, and neohesperidin). In addition, 'Siam Red Ruby' pumelo juice has high amounts of fructose, glucose, and sucrose, at 6.07, 4.52, and $2.09 \mathrm{~g} \cdot \mathrm{L}^{-1}$, respectively (Kaewsuksaeng and Sangwanangkul, 2015).

Carotenoids are considered to be the major components responsible for the attractive color of the peel and pulp in citrus fruit (Kato, 2012). The carotenoid content and composition determine the quality and appearance of fruit. The carotenoid biosynthesis pathway has been investigated in citrus fruit, and most of this metabolic 
pathway has been confirmed. Previous research revealed that carotenoid accumulation was controlled by genes involved in the carotenoid metabolic pathway (Kato et al., 2004). The changing of two geranylgeranyl pyrophosphate (GGPP) to phytoene catalyzed by phytoene synthase (PSY) is the initial step in the carotenoid metabolism pathway. Desaturation involves a functionally similar enzyme to phytoene desaturase (PDS) and $\zeta$-carotene desaturase (ZDS) converts phytoene to lycopene. The cyclization of lycopene is catalyzed by lycopene- $\beta$-cyclase (LCYb) and lycopene- $\varepsilon$-cyclase (LCYe). $\alpha$-Carotene is then converted to lutein by $\beta$ ring hydroxylase $(\mathrm{HYb})$ and $\varepsilon$-ring hydroxylase $(\mathrm{HYe})$. Moreover, lycopene may be cyclized by LCYb to produce $\beta$-carotene. $\beta$-Carotene is then hydroxylated by $\mathrm{HYb}$ to $\beta$-cryptoxanthin and zeaxanthin. Zeaxanthin is converted to all-trans-violaxanthin by zeaxanthin epoxidase (ZEP). In addition, carotenoid metabolism is closely associated with the biosynthesis of plant hormone abscisic acid (ABA); 9-cis-violaxanthin was catabolized by 9-cis-epoxycarotenoid dioxygenases (NCED) to produce ABA, as shown in Figure 1 (Kato et al., 2004; Rodrigo et al., 2004; Ma et al., 2018). The carotenoid accumulation that occurs during citrus fruit ripening is highly regulated by the coordinated expression of carotenoid biosynthesis genes, and differences in the variance of transcripts encoding biosynthetic enzymes is significantly associated with the carotenoid

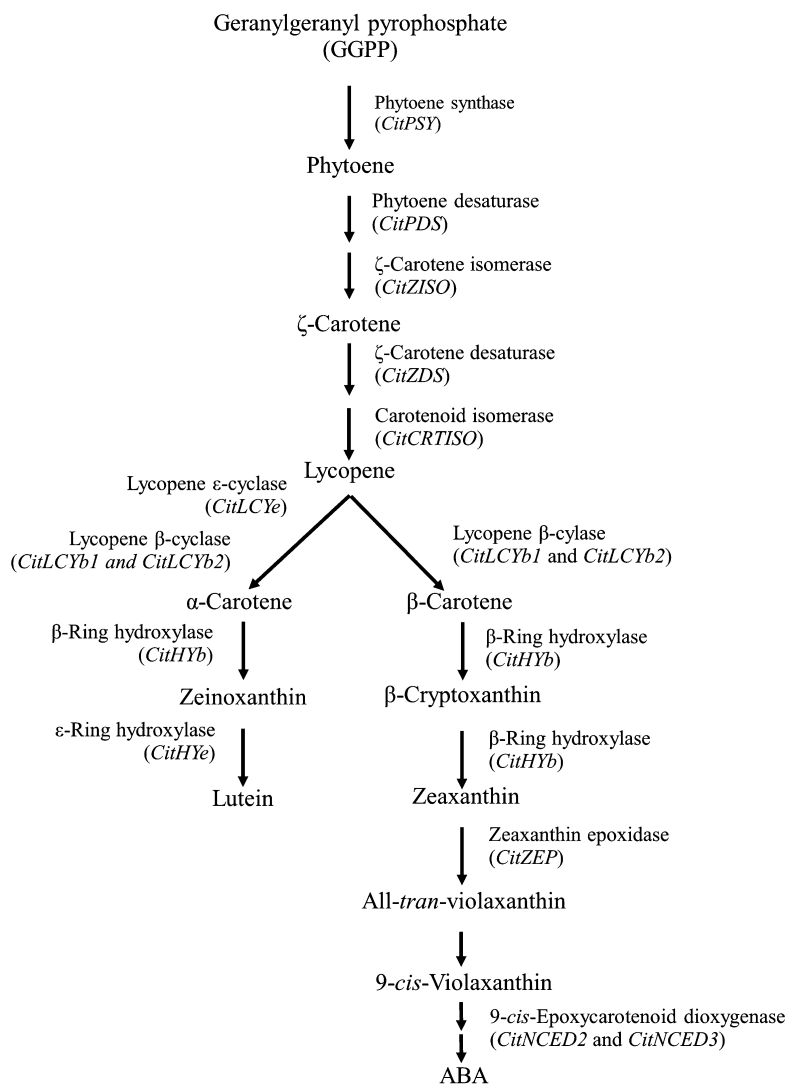

Fig. 1. The carotenoid biosynthesis pathway in citrus fruit. composition and content among varieties (Kato et al., 2004).

In citrus fruit, massive accumulation of lycopene is an unusual feature, and it has only been reported in a few species, such as Cara Cara orange (Citrus sinensis Osbeck), grapefruit (Citrus paradisi Macf.), and pumelo (Citrus grandis Osbeck) (Alquezar et al., 2008, 2009). Different from most citrus cultivars which are yellow or orange, the citrus fruit that accumulate lycopene exhibit red colors in their pulp. The red pulp of 'Siam Red Ruby' pumelo attracted the interest of producers and consumers, and research into the mechanisms of lycopene accumulation in 'Siam Red Ruby' pumelo is of great importance; it will provide a new strategy to improve 'Siam Red Ruby' pumelo's nutritional and commercial value. In the present study, carotenoid biosynthesis was investigated in a new lycopene-accumulation variety, 'Siam Red Ruby'. In addition, to elucidate the molecular mechanism of the lycopene accumulation in 'Siam Red Ruby', the gene expressions in carotenoid biosynthesis and catabolism were analyzed during fruit ripening.

\section{Materials and Methods}

\section{Plant material}

The 'Siam Red Ruby' (Citrus grandis) fruit used in this study was grown in Pakpanang district, Nakhon Si Thammarat province. The fruit was harvested during different development stages: 90 (July), 120 (August), 150 (September), 180 (October), and 210 (December) days after fruit setting. The fruit was transported to a plant science laboratory at the Faculty of Technology and Community Development, Thaksin University, Thailand. The pulp was then separated from the peel and albedo. All fruit samples were frozen immediately in liquid nitrogen, prepared for freeze-drying and stored at $-80^{\circ} \mathrm{C}$ until further use.

\section{Pulp color development and internal fruit quality}

Color measurement was performed with a colorimeter (CR300; KONIKA MINOLTA, INC., Tokyo, Japan). The CIE $1976 L^{*} a^{*} b^{*}$ color scale was adopted. The hue angle $\left(\mathrm{H}^{\circ}\right)$ was calculated as $\mathrm{H}^{\circ}=$ arctangent $\left(b^{*} / a^{*}\right)$. Color readings were taken three times at the equatorial region of each fruit.

The total soluble solid (TSS) of fruit juice was determined by using a Hand refractometer (N1; Atago Co., Ltd., Tokyo, Japan) and reported as percent soluble solids in fruit juice. The titratable acidity (TA) of fruit juice was determined by titrating $1 \mathrm{~mL}$ of fruit juice diluted 10 times with distilled water against $0.1 \mathrm{~mol} \cdot \mathrm{L}^{-1}$ $\mathrm{NaOH}$, using 1-2 drops of $1 \%(\mathrm{v} / \mathrm{v})$ phenolphthalein as an indicator of the reaction end-point. TA was expressed as percent citric acid (meq. citric acid $=0.064$ ).

\section{Extraction and determination of carotenoid content}

Carotenoids in 'Siam Red Ruby' pumelo were ex- 
tracted and measured by using high-performance liquid chromatography (HPLC) as described by (Kato et al., 2004). The contents of phytoene, $\zeta$-carotene, lycopene, $\beta$-carotene, $\beta$-cryptoxanthin, all-trans-violaxanthin, 9cis-violaxanthin, $\alpha$-carotene, and lutein in the pulp of 'Siam Red Ruby' pumelo were examined every 30 days after fruit setting during the experimental period. The carotenoid contents were converted to as $\mathrm{mg} \cdot \mathrm{kg}^{-1}$ fresh weight (FW). Carotenoid quantification was performed on three replications.

$R N A$ extraction and real-time quantitative reverse transcription-PCR (RT-PCR)

Total RNA extracted from the pulp of 'Siam Red Ruby' pumelo fruit at the different stages was analyzed using the method of Ikoma et al. (1996). Freeze-dried pulp samples $(0.5 \mathrm{~g})$ were grounded in liquid nitrogen, and $10 \mathrm{~mL}$ of phenol:chloroform:3-methyl-butanol (25:24:1) and $10 \mathrm{~mL}$ of lysis buffer containing of $0.5 \mathrm{M}$ EDTA and 1.5 M Tris-borate buffer were added, mixed and centrifuged at $3,000 \times \mathrm{g}$ for $30 \mathrm{~min}$. Two phases were separated by centrifugation and the series of steps was repeated three times. The aqueous phase was transferred to a new tube and mixed with 0.25 volume of ethanol, 0.11 volume of $5 \mathrm{M}$ potassium acetate, followed by a 1:1 ratio of chloroform:3-methy-l-butanol (49:1). Precipitation of the total RNA was done with $3 \mathrm{M} \mathrm{LiCl}$ overnight at $-20^{\circ} \mathrm{C}$. It was then pelleted by centrifugation and resuspended in DEPC-treated water. Total RNA was purified by an RNeasy Mini Kit (Qiagen, Hilden, Germany).

TaqMan MGB probes and sets of primers for CitPSY, CitPDS, CitZISO, CitZDS, CitCRTISO, CitLCYb1, CitLCYb2, CitLCYe, CitHYb, CitHYe, CitZEP, CitNCED2, and CitNCED3 were reported by Ma et al. $(2013,2016)$. The gene expression was analyzed by a StepOnePlus ${ }^{\text {TM }}$ qPCR System (Applied Biosystems) according to the manufacturer's instructions. The cycling protocol was $95^{\circ} \mathrm{C}$ for $10 \mathrm{~min}$, followed by 40 cycles at $95^{\circ} \mathrm{C}$ for $15 \mathrm{~s}$ and $60^{\circ} \mathrm{C}$ for $60 \mathrm{~s}$. The genes expression levels were calculated by using StepOnePlus ${ }^{\mathrm{TM}}$ qPCR System Software (Applied Biosystems). In the previous study, 18S ribosomal RNA was used as a reference gene in order to normalize the gene expression results. Quantitative RT-PCR was performed in three replicates.

\section{Statistical analysis}

All values are shown as the mean \pm standard error (SE) for three replicates. The data were analyzed, and Tukey's honest significant difference (HSD) test was used to compare the means at $P<0.05$. Calculations were performed using JMP software (SAS institute, Cary, NC).

\section{Results}

Color development and internal quality of 'Siam Red Ruby' pumelo

During the ripening stage, the pulp was yellow colored from 90 to 120 days after fruit setting. From 150 days after fruit setting, the pulp gradually turned red (Fig. 2). During the fruit ripening stage, the hue angle decreased rapidly (Fig. 3A), while the $a^{*}$ value of the fruit pulp increased throughout the ripening stages,

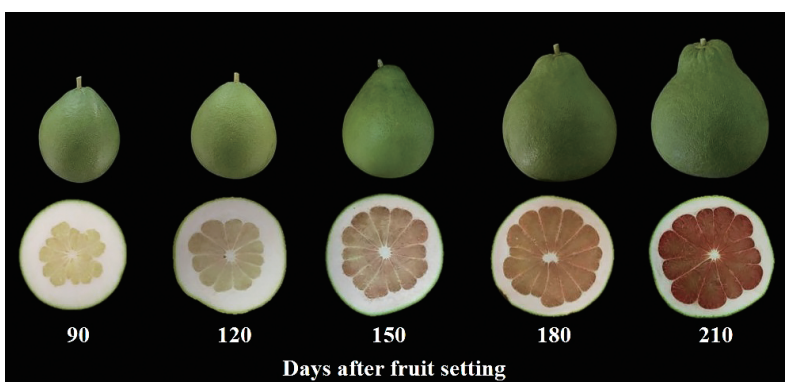

Fig. 2. Peel and pulp color changes in the 'Siam Red Ruby' pumelo fruit development stage: 90 (July), 120 (August), 150 (September), 180 (October), and 210 (December) days after fruit setting.
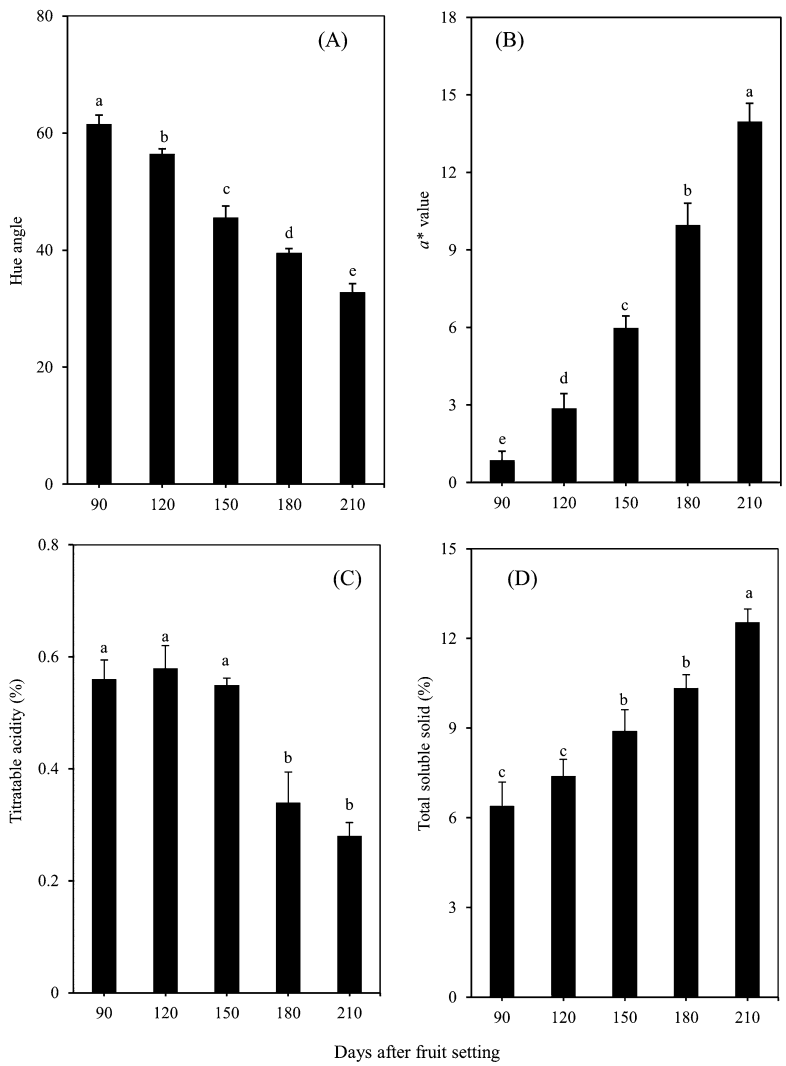

Fig. 3. Hue angle (A), $a^{*}$ value (B), titratable acidity (C), and total soluble solid (D) in the pulp of 'Siam Red Ruby' pumelo fruit during fruit development. The data shown are mean \pm standard error of three replicates. Different letters indicated significant difference by Tukey's test at $P<0.05$. 
showing higher values in the mature fruit (Fig. 3B). The changes in hue angle and $a^{*}$ values occurred in parallel with color development in the pulp. In addition, the titratable acidity (TA) was constant from 90 to 150 days after fruit setting, and then decreased significantly from 180 days after fruit setting. On the other hand, the total soluble solid (TSS) of fruit juice values increased significantly during the fruit ripening process (Fig. 3C, D).

\section{Carotenoid accumulation in the pulp of 'Siam Red Ruby' pumelo}

In this study, nine carotenoids, phytoene, $\zeta$-carotene, lycopene, $\alpha$-carotene, $\beta$-carotene, $\beta$-cryptoxanthin, alltrans-violaxanthin, 9-cis-violaxanthin, and lutein, were detected in the pulp of 'Siam Red Ruby' pumelo fruit. Among them, lycopene was the major carotenoid in the pulp, and this increased rapidly during the ripening stage, accounting for $93.26 \%$ of the total carotenoid in the maturation stage (210 days after fruit setting). During the ripening stage, $\beta$-carotene, $\beta$-cryptoxanthin, and all-trans-violaxanthin also gradually accumulated in the pulp, but their contents were much lower than lycopene, accounting for about $1.11 \%, 2.40 \%$, and $0.03 \%$ of the total carotenoid content in the mature fruit, respectively (Fig. 4). In contrast to the $\beta, \beta$-carotenoids, two $\beta, \varepsilon$ carotenoids, lutein, and $\alpha$-carotene, massively accumulated in the immature fruit (90 days after fruit setting), and then decreased rapidly during fruit ripening.
Changes in expression of carotenoid metabolic genes in the pulp of 'Siam Red Ruby' pumelo

To elucidate the mechanism of specific accumulation of lycopene in the pulp of 'Siam Red Ruby' pumelo, the expressions of carotenoid biosynthetic genes (CitPSY, CitPDS, CitZISO, CitZDS, CitCRTISO, CitLCYb1, CitLCYb2, CitLCYe, CitHYb, CitHYe, and CitZEP) and catabolic genes (CitNCED2 and CitNCED3) were analyzed by real-time quantitative RT-PCR during the fruit ripening process. As shown in Figure 5, the expression of the carotenoid biosynthetic genes CitPSY, CitPDS, CitZISO, CitZDS, and CitCRTISO was significantly up-regulated in the pulp during the ripening stage. In contrast, the expression of CitLCYb1, CitLCYb2, CitLCYe, CitHYb, and CitHYe, which were related to the biosynthesis of $\beta, \beta$ carotenoids and $\beta, \varepsilon$-carotenoids, was down-regulated during the ripening stage. In addition, the expression of two carotenoid catabolic genes, CitNCED2 and CitNCED3, decreased rapidly in the pulp during the ripening stage (Fig. 5).

\section{Discussion}

This study found significant differences in the pulp color, TSS, TA, total carotenoid content and carotenoid composition in 'Siam Red Ruby' pumelo during the development stage. During fruit development there was a difference in pulp color from 90 to 210 days after fruit
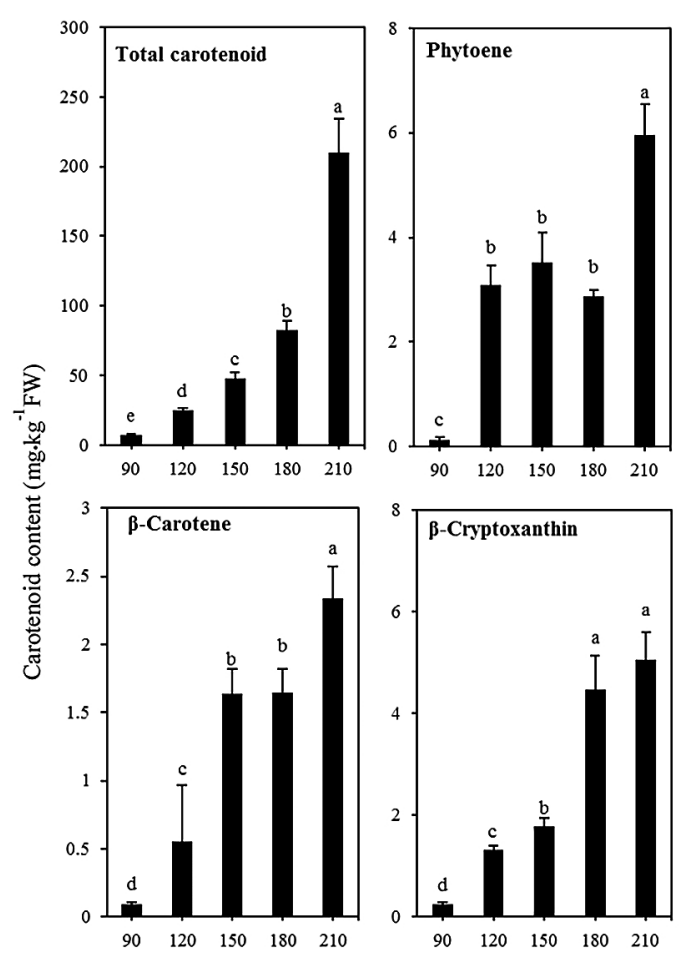
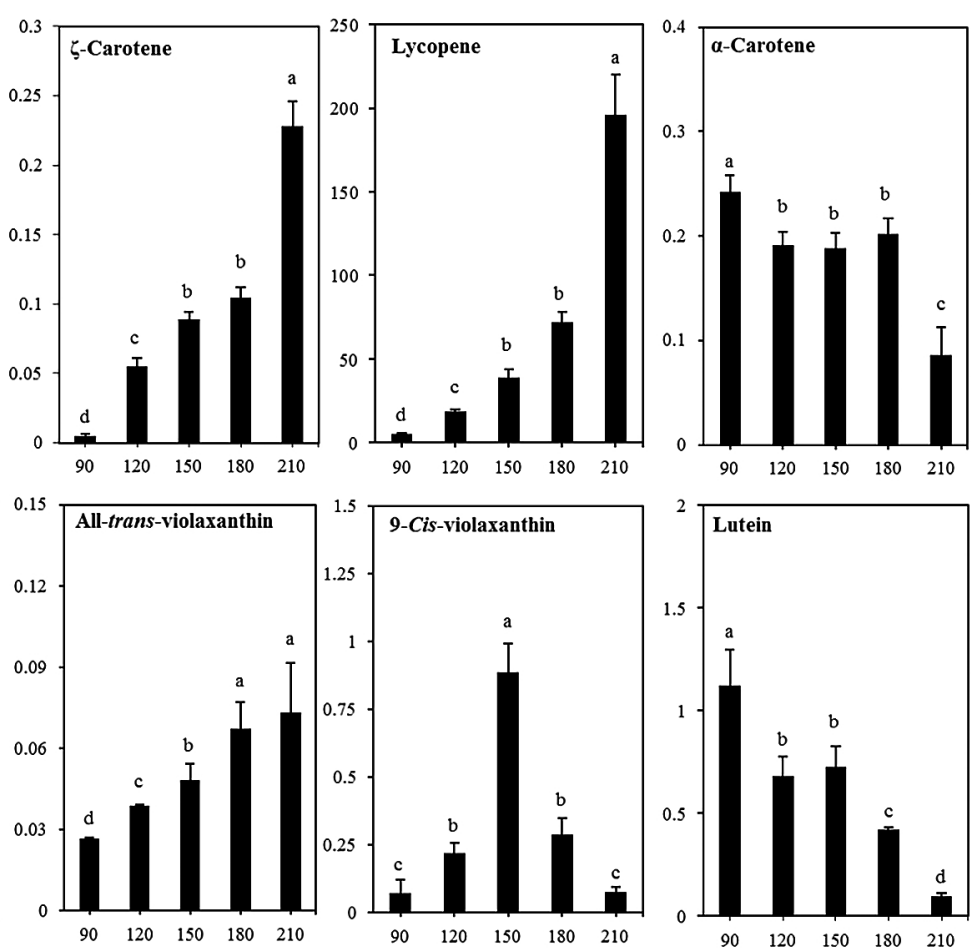

Days after fruit setting

Fig. 4. Carotenoid content and composition in fruit pulp during different development stages of 'Siam Red Ruby' pumelo. The total carotenoid content is the sum of all identified carotenoids. The data shown are mean \pm standard error of three replicates. Different letters indicated significant difference by Tukey's test at $P<0.05$. 

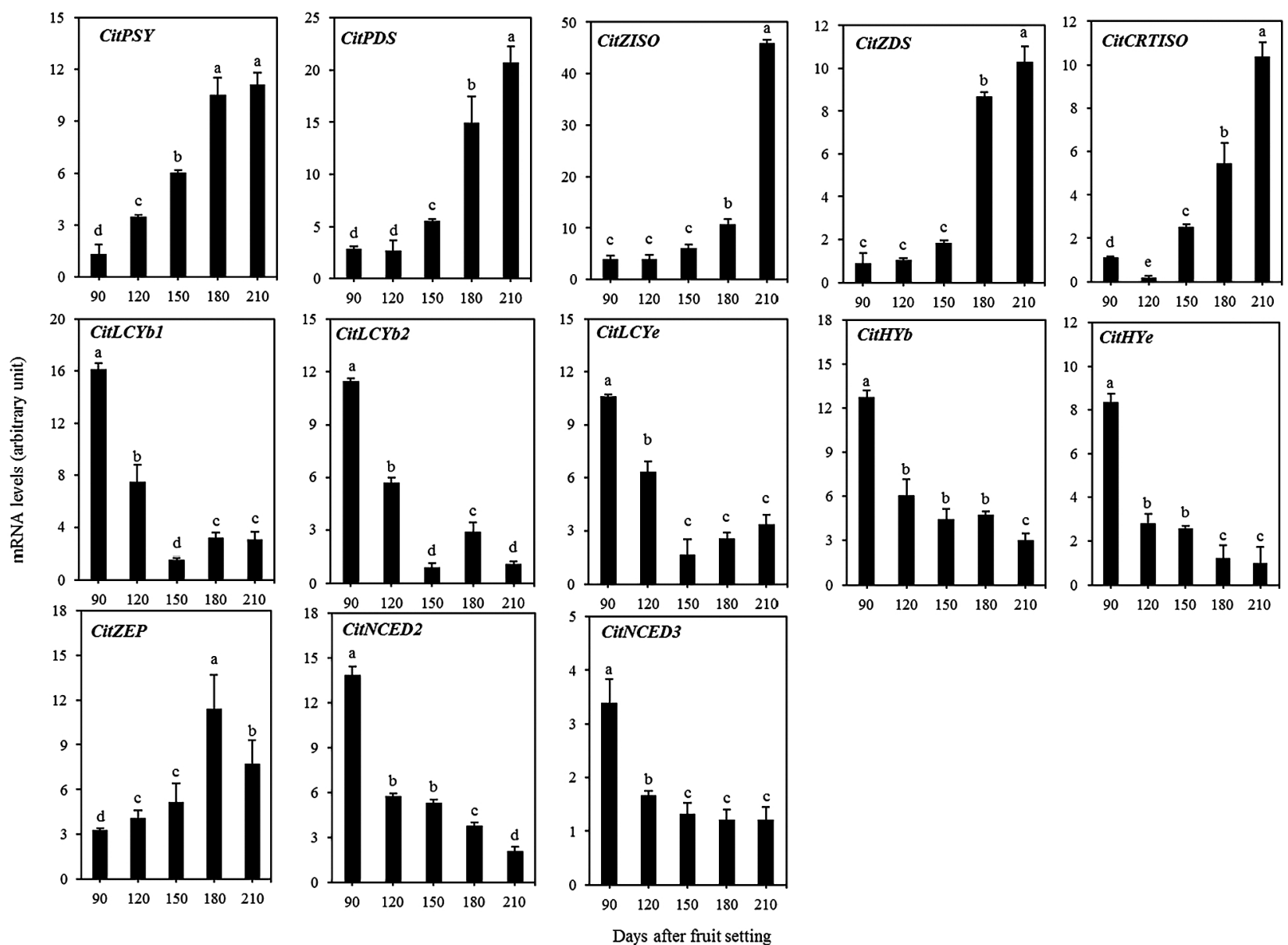

Fig. 5. Changes in the expression of carotenoid metabolism-related genes in 'Siam Red Ruby' pumelo pulp. The data shown are mean \pm standard error of three replicates. Different letters indicated significant difference by Tukey's test at $P<0.05$.

setting. The hue angle decreased sharply during this time, whereas the $a^{*}$ value increased. In addition, color development in the pulp was related to the carotenoid content, with the yellow color generally being stronger in immature fruit and reddening occurring during the ripening and development stages due to changes in the carotenoid composition. There were differences during fruit development in TSS and TA values (Fig. 3C, D). TSS increased significantly during fruit development and sugar and pigment content accumulated, while acid content decreased, in the fruit pulp. Determining the sugar/acid ratio during ripening is often used as a major postharvest maturity index. As the TSS content increases during ripening, this parameter is a very practical index of internal fruit quality. An understanding of the physiological and biochemical determinants for TSS and TA content in fruit allows for improved fruit quality during growth and pre and post-harvest, an improvement in citrus fruit sweetness and fruit acidity, characterization of physiological disorders related to TSS and TA fruit content and physicochemical composition of fruit; especially ascorbic acid, TSS and TA that depend on the maturity stage. Moreover, a high correlation between TSS and TA has been reported by
Obenland et al. (2009).

Carotenoid pigments massively accumulate in citrus fruit, and their contents and compositions are important indexes for the nutritional and commercial quality. To date, approximately 115 carotenoids have been identified in citrus (Ikoma et al., 2016). Among them xanthophylls, especially $\beta, \beta$-xanthophylls, are predominantly accumulated in the pulp of most citrus varieties. In contrast, the massive accumulation of linear carotenes in the pulp is a rare event in citrus. To date, only a few citrus varieties have been reported to accumulate lycopene as the major carotenoid in the pulp. In the present study, we investigated the carotenoid accumulation in a new lycopene-accumulating pumelo cultivar, 'Siam Red Ruby'. The results showed that the lycopene content increased rapidly during fruit ripening, and accounted for more than $93 \%$ of total carotenoid in the pulp. Moreover, in mature pulp, the lycopene content reached $195.72 \mathrm{mg} \cdot \mathrm{kg}^{-1} \mathrm{FW}$, which was much higher than previously reported in other lycopene-accumulating varieties such as Guanxi pumelo (5.84 $\left.\mathrm{mg} \cdot \mathrm{kg}^{-1} \mathrm{FW}\right)$, Hong Anliu (2.35 $\mathrm{mg} \cdot \mathrm{kg}^{-1} \mathrm{FW}$ ) (Liu et al., 2016), and Cara Cara (9 mg $\left.\cdot \mathrm{kg}^{-1} \mathrm{FW}\right)$ (Lee, 2001). In 'Siam Red Ruby', the massive accumulation of lycopene endowed the 
pulp with an attractive red color. This will make it very attractive to consumers, and also contribute to improving its nutritional value. In addition, it was interesting that the massive accumulation of lycopene only occurred in the pulp, while the flavedos was an orange color with a low level of lycopene. This suggests that carotenoid biosynthesis is independently regulated in the flavedo and pulp of 'Siam Red Ruby'. A similar phenomenon was also observed in other lycopeneaccumulating citrus varieties.

In citrus, transcriptional regulation is a key mechanism that regulates specific carotenoid accumulation in the flavedo and pulp. In the present study, the gene expression results showed that the expression of CitPSY, CitPDS, CitZISO, CitZDS, and CitCRTISO, which are related to lycopene biosynthesis, increased rapidly in the pulp of 'Siam Red Ruby' during the ripening process. In contrast, the expression of CitLCYb1, CitLCYb2, CitLCYe, CitHYb, and CitHYe, which are related to $\beta, \beta$-carotenoids and $\beta, \varepsilon$-carotenoids, decreased gradually during fruit ripening (Fig. 5). In addition, the CitPSY gene is one of the key regulatory enzymes in carotenoid accumulation and biosynthesis in citrus fruits. The changes in expression patterns of the carotenoid biosynthetic genes in 'Siam Red Ruby' were identical to those in Hong Anliu, in which increased expression of the upstream genes and decreased expression of the downstream genes was found to be the main mechanism of lycopene accumulation (Liu et al., 2016).

In plants, $\mathrm{LCYb}$ and LCYe are the two key enzymes that catalyze the cyclization of lycopene to produce $\beta, \beta-$ carotenoids and $\beta, \varepsilon$-carotenoids. In previous studies, it was reported that two LCYb genes (CitLCYbl and CitLCYb2) existed in citrus (Zhang et al., 2012). Functional analysis showed that both the enzymes of CitLCYb1 and CitLCYb2 participated in the formation of $\beta$-carotene, and when they were co-expressed with CitLCYe, $\alpha$-carotene could be produced from lycopene. During the fruit ripening process, the expression of CitLCYb1 and CitLCYb2 increased rapidly, leading to flux of linear carotenes into $\beta, \beta$-carotenoid biosynthesis in the fruit of Satsuma mandarin, Valencia orange, and Lisbon lemon (Zhang et al., 2012). In the present study, in contrast, we found that the expression of CitLCYb1 and $C i t L C Y b 2$ decreased rapidly to a low level during the fruit ripening process in 'Siam Red Ruby'. The sharp decrease in the expression of CitLCYb1, CitLCYb2, as well as CitLCYe, may restrict the biosynthesis of $\beta, \beta$-carotenoid and $\beta, \varepsilon$-carotenoids, and as a result a large amount of lycopene was accumulated in the pulp of 'Siam Red Ruby'. In citrus fruit, Lu et al. (2016) reported that the copy number of an enhancer element in $L C Y b 1$ promoters was different among different species, possibly affecting the $L C Y b 1$ expression. To date, however, the mechanisms that regulate the expression of carotenoid biosynthetic genes are still to be fully elucidated in plants. In the future, research into the transcriptional regulatory mechanism of carotenoid biosynthetic genes will be of great importance to further elucidate the massive accumulation of lycopene in 'Siam Red Ruby'. In conclusion, the carotenoid metabolism was investigated in the pulp of 'Siam Red Ruby'. The results showed that the lycopene content increased rapidly during the fruit ripening process, and the massive accumulation of lycopene in the mature fruit led to an attractive red color in the pulp. The gene expression results showed that the increased expression of the upstream genes and decreased expression of downstream genes in the carotenoid biosynthetic pathway were the main mechanism of lycopene accumulation. The present results will contribute to further elucidating the mechanisms of carotenoid accumulation in 'Siam Red Ruby' pumelo, which will provide new insights into improving its nutritional and commercial value.

\section{Literature Cited}

Alquezar, B., M. J. Rodrigo and L. Zacarías. 2008. Regulation of carotenoid biosynthesis during fruit development in the redfleshed orange mutant Cara Cara. Phytochemistry 69: 19972007.

Alquezar, B., L. Zacarias and M. J. Rodrigo. 2009. Molecular and functional characterization of a novel chromoplast-specific lycopene $\beta$-cyclase from Citrus and its relation to lycopene accumulation. J. Exp. Bot. 60: 1783-1797.

Department of Agriculture Extension (DOAE). 2019. The agricultural statistics of Thailand the crop year [Online]. $<$ http:// www.doae.go.th>.

Ikoma, Y., H. Matsumoto and M. Kato. 2016. Diversity in the carotenoid profiles and the expression of genes related to carotenoid accumulation among citrus genotypes. Breed. Sci. 66: 139-147.

Ikoma, Y., M. Yano, K. Ogawa, T. Yoshioka, Z. C. Xu, S. Hisada, M. Omura and T. Moriguchi. 1996. Isolation and evaluation of RNA from polysaccharide-rich tissues in fruit for quality by cDNA library construction and RT-PCR. J. Japan. Soc. Hort. Sci. 64: 809-814.

Kaewsuksaeng, S. and P. Sangwanangkul. 2015. Bioactive compound contents in citrus family locally cultivated in southern Thailand. Khon Kaen Agr. J. 43: 799-804 (In Thai with English abstract).

Kaewtubtim, M. and M. Issarakraisila. 2011. Effects of nitrogen and zinc on fruit quality of pummelo cv. Tubtim Sayam. Proceedings of the Commission on Higher Education Congress IV University Staff Development Consortium. 14-16 (In Thai with English abstract).

Kato, M. 2012. Mechanism of carotenoid accumulation in citrus fruit. J. Japan. Soc. Hort. Sci. 81: 219-233.

Kato, M., Y. Ikoma, H. Matsumoto, M. Sugiura, H. Hyodo and M. Yano. 2004. Accumulation of carotenoids and expression of carotenoid biosynthetic genes during development in citrus. Plant Physiol. 134: 824-837.

Lee, H. S. 2001. Characterization of carotenoids in juice of red navel orange (Cara Cara). J. Agric. Food Chem. 49: 25632568.

Liu, W., Q. Ye, X. Q. Jin, F. Q. Han, X. Z. Huang, S. G. Cai and L. Yang. 2016. A spontaneous bud mutant that causes lycopene and $\beta$-carotene accumulation in the juice sacs of the parental Guanxi pummelo fruits (Citrus grandis (L.) 
Osbeck). Sci. Hortic. 51: 349-353.

Lu, S., Y. Zhang, X. Zheng, K. Zhu, Q. Xu and X. Deng. 2016. Isolation and functional characterization of a lycopene $\beta$ cyclase gene promoter from citrus. Front. Plant Sci. 7: 13671380 .

Ma, G., L. C. Zhang, A. Matsuta, K. Matsutani, K. Yamawaki, M. Yahata, A. Wahyudi, R. Motohashi and M. Kato. 2013. Enzymatic formation of $\beta$-citraurin from $\beta$-cryptoxanthin and zeaxanthin by carotenoid cleavage dioxygenase 4 in the flavedo of citrus fruit. Plant Physiol. 163: 682-695.

Ma, G., L. Zhang, W. Yungyuen, Y. Sato, T. Furuya, M. Yahata, K. Yamawaki and M. Kato. 2018. Accumulation of carotenoid in a novel citrus cultivar 'Seinannohikari' during the fruit maturation. Plant Physiol. Bioch. 129: 349-356.

Ma, G., L. Zhang, W. Yungyuen, W. Tsukamoto, N. Iijima, M. Oikawa, K. Yamawaki, M. Yahata and M. Kato. 2016. Ex- pression and functional analysis of citrus carotene hydroxylases: unravelling the xanthophyll biosynthesis in citrus fruits. BMC Plant Biol. 16: 148. DOI: 10.1186/s12870-0160840-2.

Obenland, D., S. Collin, B. Mackey, J. Sievert, K. Fjeld and M. L. Arpaia. 2009. Determinants of flavor acceptability during the maturation of navel oranges. Postharvest Biol. Technol. 52: 156-163.

Rodrigo, M. J., J. F. Marcos and L. Zacarías. 2004. Biochemical and molecular analysis of carotenoid biosynthesis in flavedo of Orange (Citrus Sinensis) during fruit development and maturation. J. Agric. Food Chem. 52: 6724-6731.

Zhang, L., G. Ma, Y. Shirai, M. Kato. K. Yamawaki, Y. Ikoma and H. Matsumoto. 2012. Expression and functional analysis of two lycopene $\beta$-cyclases from citrus fruits. Planta 236: $1315-1325$. 